\title{
Premier inventaire faunistique des Chironomidés (Diptera, Chironomidae) du Maroc
}

\author{
A. Azzouzi ${ }^{1}$ \\ H. Laville ${ }^{2}$
}

Mots clés : Diptera, Chironomidae, faunistique, biogéographie, Maroc.

65 espèces de Chironomidés ont été inventoriées du Maroc à partir de l'analyse des données de la littérature parue entre 1955 et 1986 . Des récoltes plus récentes dans divers oueds traversant les villes de Fès, Meknès, Khenifra, Marrakech, nous ont permis de porter à 134 le nombre des Chironomidés recensés à présent sur le territoire marocain. Ils se répartissent ainsi : 16 Tanypodinae, 8 Diamesinae, 42 Orthocladinae, 45 Chi ronomini, 23 Tanytarsini. 11 espèces (8,2\%) sont d'origine éthiopienne et 6 espèces sont indigènes d'Afrique du Nord : Rheopelopia n. sp.. Telopelopia maroccana, Rheomus alatus, Rheomus yahiae, Stempellina almi, Virgatanytarsus ansatus.

First faunistic inventory of chironomids (Diptera, Chironomidae) of Morocco.

Keywords : Diptera, Chironomidae, faunistic, biogeography, Morocco.

65 species of chironomids have been recorded from Morocco according to an analysis of data from the literature between 1955 and 1986. More recent collections from various rivers going through the towns of Fes, Meknes, Khenifra, Marrakech, have now increased to 134 the number of chironomid species recorded from Moroccan territory. They are distributed thus : 16 Tanypodinae, 8 Diamesinae, 42 Orthocladinae, 45 Chironomini, 23 Tanytarsini. 11 species (8,2 \%) are of Ethiopian origin and 6 species are indigenous to North Africa : Rheopelopia n. sp., Telopelopia maroccana, Rheomus alatus, Rheomus yahiae, Stempellina almi, Virgatanytarsus ansalus.

\section{Introduction}

Jusqu'à présent, très peu d'études ont été consa. crées aux Chironomidés d'Afrique du Nord où seulement 45 espèces sont répertoriées dans la Limnofauna Europaea (Fłttkau \& Reiss 1978).

Au Maroc, l'analyse des données de la littérature, antérieures à 1986 nous a permis de recenser quelque 65 espèces signalées, pour la plupart, des régions côtières du Nord entre Rabat et la frontière algérienne (Berkane) et de quelques sites des montagnes du Haut Atlas. A partir de ces données et de récoltes récentes recueillies dans divers oueds traversant les villes de Fès, Meknès, Khenifra et

1. Laboratoire d'Hydrobiologie, Université Cadi Ayyad, Faculté des Sciences, Boulevard de Safi, Marrakech, Maroc.

2. Laboratoire d'Hydrobiologie, UA 695 C.N.R.S., Universite P. Sabatier, 118 route de Narbonne, 31062 Toulouse Cédex, France.
Marrakech, nous présentons le premier inventaire faunistique des Chironomidés actuellement connus du Maroc.

\section{Citations antérieures à 1986}

La plupart des données bibliographiques, antérieures à 1986, qui signalent la présence de Chironomidés au Maroc, restent fragmentaires, les collections provenant le plus souvent d'entomologistes non spécialistes dont les récoltes sont dispersées dans le temps et dans l'espace.

Cependant, ce sont la zone littorale du Nord Rabat, Kénitra, Larache, Tanger, Tetouan, Berkane - et les montagnes du Haut Atlas qui ont été surtout visitées.

Reiss (1977) établit la première importante liste de 37 Chironomidés, dont 6 espèces nouvelles, recensées sur le territoire marocain. Il s'agit d'une grande 
Tableau I : Inventaire 1987 des Chironomidés du Maroc. Les espèces nouvellement citées depuis 1986 sont précédées d'un astérisque. Les nombres renvoient aux références bibliographiques.

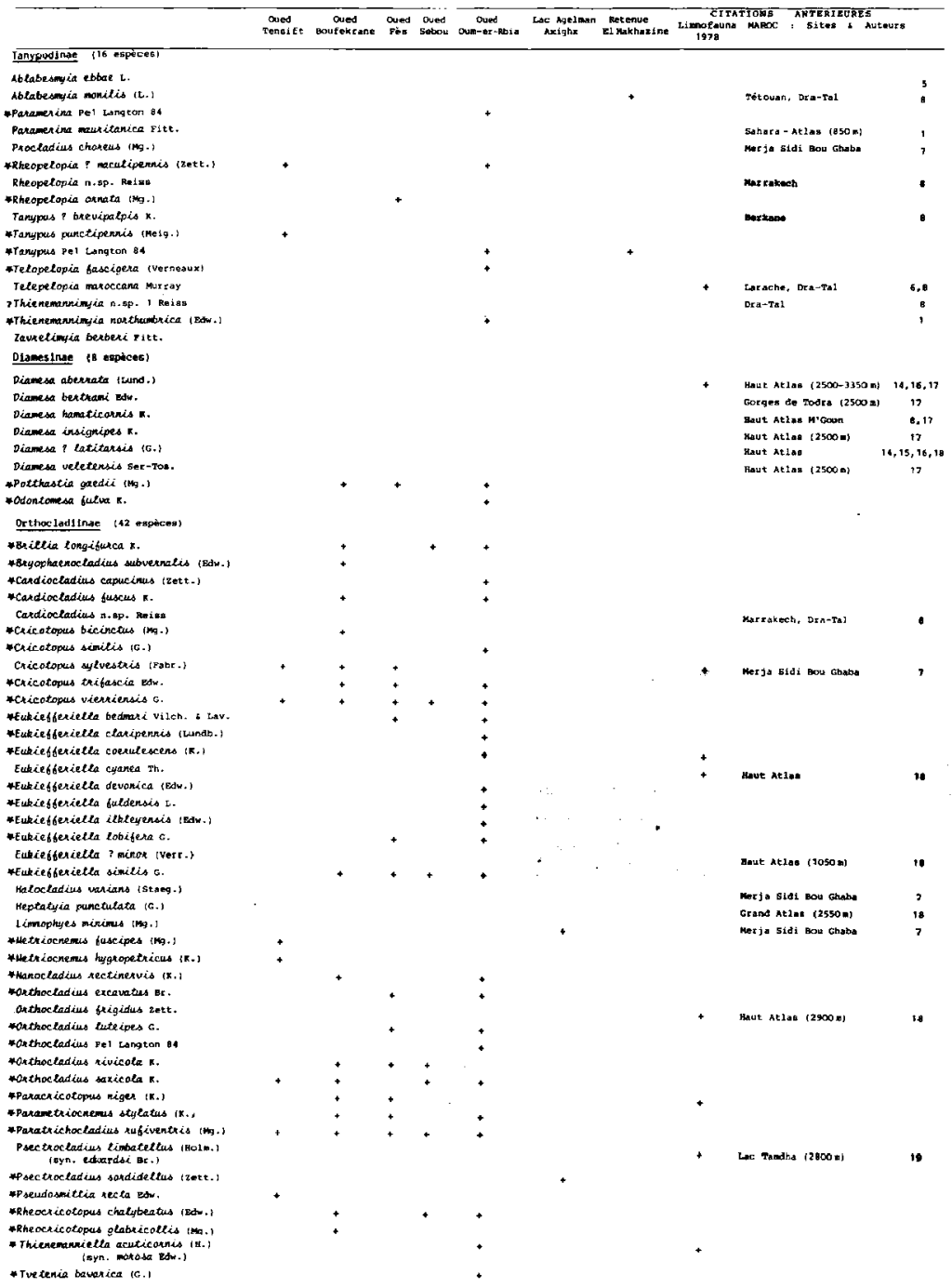




\begin{tabular}{|c|c|c|c|c|c|c|c|c|c|c|}
\hline & Denest & cues & wad & aved & $\underset{\text { and }}{\text { ander-ents }}$ & 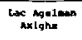 & extanue & $\begin{array}{l}\text { Crish } \\
\text { timofours } \\
\text { 397" }\end{array}$ & 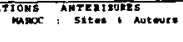 & \\
\hline \multicolumn{11}{|l|}{ Chitronomint 145 especess } \\
\hline 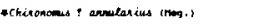 & - & & & & & & & & & \\
\hline 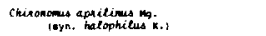 & & & & & & & & & Merjo Hiak sou Grabs & 7 \\
\hline Chitonomes calinterus $x$. & & & & & & & & + & 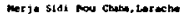 & he 7.8 \\
\hline Cheronomis berides stx. & & & & & & & & & Mer Jo sidi bou Grabe & , \\
\hline thironomes pigen str. & & & & & & & & & Mer ja sidi sou chata & 7 \\
\hline Chironomas plumodes L. & & & & & & & * & & Ner ga sioi and chaten & $?$ \\
\hline Chilenomes niparies n. & & + & - & + & & & & & Merje sldi sou ands & , \\
\hline Chironomes satimarius $\mathrm{k}$. & & & & & & & & + & Merje slat sou chates & , \\
\hline *eryptochinanomes sabtratus $\mathrm{n}$. & - & - & - & - & + & & & & & \\
\hline Ficroterdipes paltidic arnis $c$. & & • & & & & & $\cdot$ & & & \\
\hline $\begin{array}{l}\text { Dicratendipes patasimanis K. } \\
\text { - Glypototendipes pallens iny. }\end{array}$ & & & & & & & + & + & Leracke & \$ \\
\hline 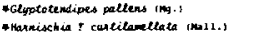 & & & - & + & & & & & & \\
\hline 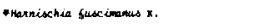 & & + & & & & & + & & & \\
\hline Kief betulus tendipediformis $c$. & & & & & & & & + & Merja siel Bow fibetu, Tétoulan & in 7.8 \\
\hline nicrochinonowes den inae rros. & & & & & & & & & Mer je sidi best chand, Rabat & 7.8 \\
\hline Wicrockironomes lendili (K.) & & & & & & & & & 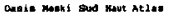 & 30 \\
\hline Hectatendipes difsinis iRdu.) & & & & & & & & + & Dera-Ta2 & a \\
\hline mickotendipes pedellus (a* ceor) & & & & & & & & + & 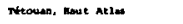 & 8 \\
\hline Parsechitonomes paritis (welk, & & & & & & + & & + & Lareme & s \\
\hline Parectedopelma lamerata $x$. & & & & & & & & + & Dre-7nel & - \\
\hline 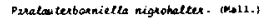 & & - & - & + & & & & & & - \\
\hline 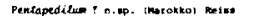 & & & & & & & & & $D * z-T a 1$ & - \\
\hline Polupedilum acifer zownes & & + & - & * & & & - & ? & Dra-tal & 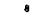 \\
\hline 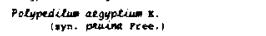 & & & & & & & - & & $\begin{array}{l}\text { Larachi, Ma rrakech, corget } \\
\text { de roudre }\end{array}$ & 0, \\
\hline aprelypedilum bicrenatum $x$. & & & & + & & & & & & \\
\hline Polypedilum convictum iwalk.) & & + & & & & & & * & Lormote & - \\
\hline Polypedilua cultelletion f. & & & & & + & & & + & & \\
\hline 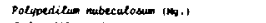 & & & & + & & & & + & Ntowen & - \\
\hline - Polypedileun nubens (Edu-) & & & & + & & & & & & \\
\hline Polypedicum mubilee (skuse: & & & & & & & & & & 3 \\
\hline Fpolupeditum pedestre ing-] & & * & & & & & & & & : \\
\hline Polypeditum phatea ox. & & & & & & & & - & Larache, Rasat, Mer fu Sid1 B. Gh & G. $.7,8$ \\
\hline polypeditur pultur izete.] & * & - & & & * & & & & & \\
\hline Tpolypedilen uncinction G. & & + & - & & & & & & & \\
\hline Polypedichum dealaemum (scht.) & & & & & & & & + & $7 x+-T+1$ & a \\
\hline oRheomes alsters Loville inales & - & & & & & & & & & \\
\hline 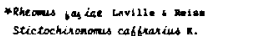 & & & 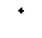 & & & & & & & - \\
\hline astictachironomis metipennis in. & & & & + & & & & & & \\
\hline Stictocheromomus pictulus (n.) & & & & & & & & + & Lareche & . \\
\hline *stictochinanomes seciclicus (rabz:) & + & & & & & & & & & \\
\hline Seictochiromomes n.sp. Remile. & & & & & & & & & $x \cdot h=10$, Dra-Ta1 & - \\
\hline Exerochiromones remolatis $x$. & & & $\bullet$ & . & & & & & & \\
\hline Ianytars!n1 123 especes! & & & & & & & & & & \\
\hline 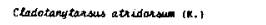 & & & & & & & & & Marja sidt Bow Chaba & , \\
\hline Clactocany tatsus mancus (Matk-) & & & & & & & & & 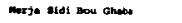 & ? \\
\hline * Cladosany tansus pes Langton in & & & & - & 4 & & & & & \\
\hline 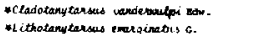 & + & & & & + & & & & & \\
\hline 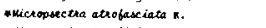 & & & & + & + & & & + & & \\
\hline Hictomsectio tenclsula (G.) & & & & & & & & + & Le kroniche & - \\
\hline Peratanyzensus inopertens Malk.; & & & & & & & & - & treosen & 0.12 \\
\hline 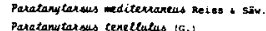 & & & & & & & & & 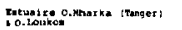 & 8,12 \\
\hline $\begin{array}{l}\text { Patazanytaresus ceneltuless (6.) } \\
\text { Rheotanytar sus mescicole } \mathrm{K} \text {. }\end{array}$ & & & & & & & & t & & 0,12 \\
\hline * Rectanytarsus neissi 1. & & + & & & + & & & + & Lereche, nort takech, Dre-tal & \\
\hline Rheoranifarsics senoci $t$. & & + & + & + & + & & & - & Tetoosen & • \\
\hline $\begin{array}{l}\text { - Stempetlina stai ar. } \\
\text { Tanstarsus : tre vipalpis a. }\end{array}$ & & + & & & & & & - & & \\
\hline 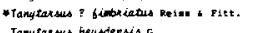 & + & & + & + & & & & * & Markan. & - \\
\hline Tanytaroses heusdensis $\mathrm{C}$. & & & & & & & & + & thed-Tal & 8.11 \\
\hline Tenytatseses horeni a. & • & & & & & & & + & Teroven, Laterche Menitt: & $7,8,1+$ \\
\hline Tanytertsus n.ap. ICreticun! Reins" & & & & & & & & & Berkane & $\mathbf{a}$ \\
\hline 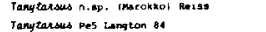 & & & & & & & & & $D e s-m i$ & 2,0 \\
\hline 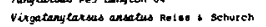 & & & & & & & & & & \\
\hline 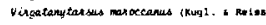 & & & & & + & & & + & $\begin{array}{l}\text { Havt At 1el } \\
\text { Ora-Tel }\end{array}$ & $\begin{array}{ll}13 \\
10,13\end{array}$ \\
\hline
\end{tabular}


majorité d'espèces de milieux stagnants : Tanypodinae (5) et Chironominae (30).

Rhamdani \& Tourenq (1982) signalent 18 espèces, dont 14 Chironominae, dans différents milieux sta. gnants de la Merja de Sidi Boughaba, proche de Rabat.

Serra-Tosio (1983) cite 4 Diamesinae du Haut Atlas, les citations antérieures de Diamesa latitarsis lui paraissant douteuses et peu probables.

La plupart des autres citations d'especes marocaines sont disséminées dans quelques révisions génériques ou dans des travaux de descriptions d'espèces nouvelles dont les holotypes ont été trouvés au Maroc.

Voici la liste des travaux qui nous ont permis de dresser le premier recensement des Chironomidés signalés depuis ces trente dernières années. Ils sont récapitulés dans l'ordre chronologique de parution : Vaillant (1955), Wülker (1959), Fittkau (1962), SerraTosio (1967), Kügler \& Wool (1968), Saether (1968), Lehmann (1970), Reiss \& Fittkau (1971), Kügler \& Reiss (1973), Serra-Tosio (1973), Reiss (1977), Murray (1980), Lehmann (1981), Reiss \& Säwedal (1981), Rhamdani \& Tourenq (1982), Serra-Tosio (1983), Reiss \& Church (1984), Reiss $(1985,1986)$.

Dans le tableau I ces références sont numérotées de 1 à 19 en suivant l'ordre alphabétique des travaux cités en bibliographie.

Dans les références antérieures à Reiss (1977) nous n'avons retenu que les taxons identifiés à l'espèce.

Les citations en Afrique du Nord de la Limnofauna Europaea 1978 et les citations au Maroc antérieures à 1986 sont reportées dans les 3 dernières colones de ce tableau.

\section{Nouvelles récoltes}

Sur l'ensemble du réseau hydrographique du Maroc, l'un de nous (A. Azzouzi) a échantillonné 4 cours d'eau ou oueds à deux-trois périodes en 1986 et 1987 à l'aide de filets à dérive : trois oueds de plaine - l'O. Boufekrane, l'O. Fès, l'O. Sebou et un oued de moyenne montagne - l'O. Oum-er-Rbia- dans le Moyen Atlas.

Nous avons également inclus dans cette étude des récoltes plus anciennes provenant de l'Oued Tensift au niveau de Marrakech (G. Masson leg.). L'ensemble des résultats de nos récoltes est récapitulé dans les cinq premières colonnes du tableau I. Quelques milieux stagnants ont été prospectés au cours d'une seule visite : I retenue, en août 1987 et 2 lacs naturels, en décembre 1987. Les espèces nouvellement citées du Maroc sont précédées d'un astérisque.

\subsection{Oued Tensift et Oukaimeden (Marrakech)}

14 especes provenant de la Palmeraie de la ville de Marrakech $(800 \mathrm{~m}), 6$ sur la rive Sud de l'Oued Tensift et 3 espèces, Metriocnemus fuscipes, $M$. hygropetricus, Ch. annularius, capturées en mai 1981 dans une station du Haut Atlas, à Oukaimeden $(2500 \mathrm{~m})$ ont été identifiées sur des imagos $\sigma 0$ capturés en vol.

Sur ces 17 espèces, 14 sont nouvelles pour le Maroc. Signalons dans l'O. Tensift la récolte, le 15.09.78, de la nouvelle espèce Rheomus alatus $n$. sp. appartenant à un nouveau genre récemment décrit (Laville \& Reiss, sous presse).

\subsection{Oued Boufekrane (Meknès)}

Sur l'Oued Boufekrane ( $28 \mathrm{~km}$ ), petit affluent rive gauche de l'Oued Sebou, 5 stations entre $750 \mathrm{~m}$ et $340 \mathrm{~m}$ furent échantillonnées, en amont et en aval de la ville de Meknès.

35 taxons - 32 identifiés à l'espèce - ont pu être recensés sur la base de 3 séries de dérives effectuées en décembre 85 , avril et août 86 .

\subsection{Oued Fès et Oued Sebou (Fès)}

Les 4 stations échantillonnées sur l'Oued Fès, petit affluent (22 km) de l'Oued Sebou, sont situées en amont de la ville de Fès à une altitude approximative de $380 \mathrm{~m}$.

Sur les 3 stations de l'Oued Sebou, 2 sont situées en amont $(220 \mathrm{~m}), 1$ en aval $(180 \mathrm{~m})$ de la confluence de l'Oued Fès.

Dans l'ensemble de ces 7 stations, 40 taxons ont été dénombrés : 29 espèces dans l'O. Fès et 18 espèces dans l'O. Sebou.

Parmi les espèces intéressantes de ce peuplement signalons:

- Eukiefferiella bedmari n. sp. Vilchez \& Laville (1987) ;

- Rheomus yahiae n. sp. Laville \& Reiss (sous presse) : espece d'un nouveau genre dont le locus 
typicus se situe au Maroc à S. Yahia Boka et qui est également connue de Tunisie :

- Polypedilum acifer Townes : espèce à répartition holarctique signalée des U.S.A. - Michigan (Townes 1945), des Carpathes (Fittkau \& Reiss 1978), de Roumanie (Albu 1980), de Yougoslavie et de Grèce (Reiss 1977).

\subsection{Oued Oum-er-Rbia (Khenifra)}

L'Oumer-Rbia (555 km) est avec l'Oued Sebou (545 km) l'un des deux plus longs oueds marocains is sus du Moyen Atlas.

3 stations ont été prospectées dans l'épirhitral entre la Source $(1500 \mathrm{~m})$ et l'aval de Khenifra (1280 $\mathrm{m})$ en août 86 et janvier 87 .

Sur les 41 espèces identifiées, 18 sont nouvelles pour la faune marocaine.

Signalons comme espèces intéressantes:

- Eukiefferiella bedmari Vilchez \& Laville

- Lithotanytarsus emarginatus $\mathbf{G}$.

- Rheotanytarsus ringei Lehm.

- Virgatanytarsus maroccanus (Kugl. \& Reiss).

Le tableau II récapitule les peuplements Chironomidiens des Oueds Boufekrane, Fès + Sebou et Oum-er-Rbia. Avec 24 espèces, l'oued de moyenne montagne Oum-er-Rbia présente le peuplement avec la plus grande diversité spécifique au niveau des Orthocladiinae, moins nombreux dans les oueds de plaine, Boufekrane (16) ou Fès + Sebou (15), où les Chironominae prédominent (22).

Tableau II : Répartition par sous-familles et tribus des Chironomidés dans les 4 oueds marocains nouvellement prospectés.

\begin{tabular}{lccc}
\hline & $\begin{array}{c}\text { Oued } \\
\text { Boufekrane }\end{array}$ & $\begin{array}{c}\text { Oueds } \\
\text { + Sebou }\end{array}$ & $\begin{array}{c}\text { Oued } \\
\text { Oum-er-Rbia }\end{array}$ \\
\hline Tanypodinae & 1 & 2 & 5 \\
Diamesinae & 1 & 1 & 1 \\
Orthocladiinae & 16 & 15 & 24 \\
Chironomini & 10 & 16 & 7 \\
Tanytarsini & 5 & 6 & 7 \\
Total & 33 & 40 & 44 \\
\hline
\end{tabular}

\subsection{Milleux stagnants}

Dans le réseau hydrographique du Moyen Atlas, deux lacs naturels, les lacs Ouiouane $(1635 \mathrm{~m})$ et
Agelman Axighx (1500 m) et le lac de retenue de El Makhazine $(357 \mathrm{~m})$ furent prospectés à une seule date (décembre 86).

Le lac Ouiouane se situe dans le Causse del Hamman au nord des sources de l'Oum-er-Rbia; le lac Agelman Agighx est situé dans le Causse d'Ajdir au sud des sources de l'Oum-er-Rbia. La retenue El Makhazine barre l'Oued Loukkos qui prend sa source à $1400 \mathrm{~m}$ d'altitude et se jette dans l'atlantique au nord de Larache.

Sur 15 taxons recensés dans ce matériel, 3 espèces Psectrocladius sordidellus, Dicrotendipes pallidicornis et Glyptotendipes pallens s'avèrent nouvelles pour le Maroc.

Les récoltes dans Agelman Axighx et El Makhazine sont notées dans les colonnes 6 et 7 du tableau I. Ps. sordidellus est la seule espèce identifiée du lac Ouiouane.

\section{Localisation géographique des sta- tions marocaines}

Tous les sites de récoltes de Chironomidés prospectés ces trente dernières années, entre 1955 et 1987, sont présentés sur la carte du Maroc (fig. 1).

Nous récapitulons ci-après les noms des collectionneurs avec leurs années et leurs sites respectifs de prospections. Les régions où les récoltes furent le plus intensives sont signalées en caractères gras.
1. Rabat
Choumara 1968
M. Ramdani 1978 et 1979 : eaux sta- gnantes de la Merja de Sidi Boughaba.
2. Kenitra
3. Larache Choumara 1968.
F. Ringe 1967 : estuaire de l'Oued Lou- hos, $15 \mathrm{~km}$ SE Larache.
Choumara 1968 : environs de Larache.
A. Azzouzi 1986 : retenue El Makhazine (357 m).
4. Tanger
5. Tetounan
6. Berkane
7. Meknès
F. Ringe 1967 : Mare Kenna, estuaire de l'Oued Mharkar, $25 \mathrm{~km}$ SO Tanger. Choumara 1968 : environs de Tetouan. Choumara 1968 : environs de Berkane. A. Azzouzi 1986,84: Oued Boufekrane (350-750 $\mathrm{m})$.
8. Fès A. Az7ouzi 1986, 87 : Oued Fès (400 m) et Oued Sebou (180-221 m).
9. Moyen Atlas Kenifra: A. Azzouzi 1987: Amont Oued Oum-er-Rbia (1500-1280 m); lac Ouiouane $(1635 \mathrm{~m})$; lac Agelman Axighx (1500 m). 


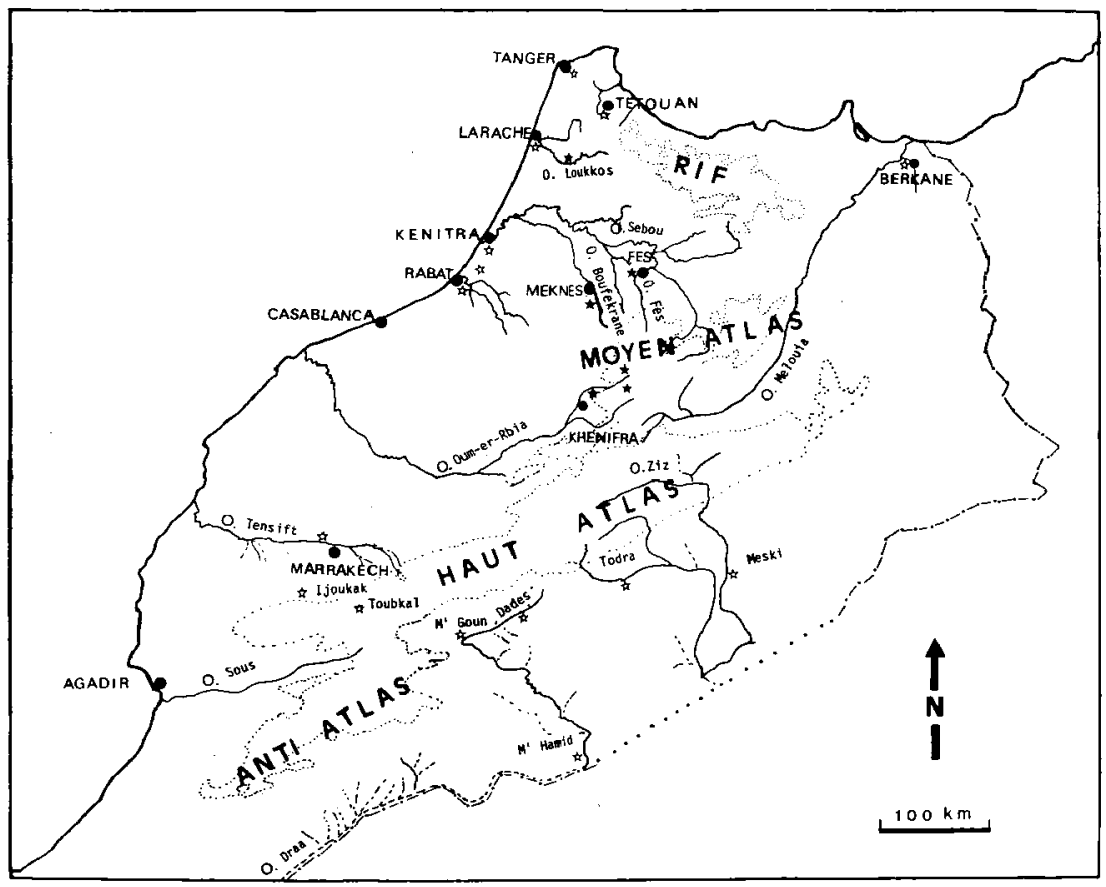

Fig. 1 : Carte de localisation des principaux sites de recolles de Chironomidés avant 1986 (eloile blanche) et apres 1986 (étoile noire)

10. Haut Atlas G. Fontaine 1979: Gorges de Todra $(1400 \mathrm{~m})$.

F. Aver \& K. Werner 1979 : Gorges de Todra et Gorges de Dades ( $2500 \mathrm{~m}$ ). Choumara 1968 : Dra-Tal (2500-3000 m).

F. Ringe 1966: N Hamid Dra-Ta1.

F. Vaillant 1954: Source de M'Goun $(2500 \mathrm{~m})$.

F. Vaillant 1954 : Lac Tamdha (2800 m). Choumara 1968 : Tissint M. Dra.

J. Lehmann \& F. Ringe 1967 : Mare près Ijoukak.

11. Sud Haut

Atlas

G. Fontaine 1979 et F. Aver \& K. Werner. 1979 : Oasis Meski (1160 m).

12. Marrakech Choumara 1968.

G. Masson 1979, 81 : Palmeraie Oued Tensift $(800 \mathrm{~m})$.

\section{Marrakech G. Masson 1979: Massif du Toubkal Oukaimeden $(2500 \mathrm{~m})$}

On peut remarquer que les milieux actuellement les mieux connus se répartissent en 3 groupes :

a) Plans d'eau des environs de Rabat ;

b) Oueds des montagnes du Moyen Atlas (Khenif ra) entre $1280 \mathrm{~m}$ et $1500 \mathrm{~m}$ et du Haut Atlas entre $1400 \mathrm{~m}$ et $3000 \mathrm{~m}$;

c) Oueds de plaine des environs de Fès, Meknès et Marrakech entre $180 \mathrm{~m}$ et $750 \mathrm{~m}$.

\section{Conclusion}

Une première liste de 65 espèces de Chironornidés connus du Maroc a été établie à partir des données de la littérature parue entre 1955 et 1986. 
Tableau III : Répartition des Chironomidés du Maroc dans les différentes sous-familles ou tribus recensés globalement avant 1986, et après 1986 dans les 3 principaux sites de récolte.

\begin{tabular}{|c|c|c|c|c|c|c|}
\hline Nb. espèces & Tanypodinae & Diamesinae & Orthocladiinae & Chironomini & Tanytarsini & Total \\
\hline Littérature avant 1986 & 9 & 6 & 9 & 27 & 14 & 65 \\
\hline $\begin{array}{l}\text { O. Tensift }+ \\
\text { Oukaimeden }\end{array}$ & 2 & & 6 & 5 & 2 & 15 \\
\hline $\begin{array}{l}\text { O. Boufekrane, } \\
\text { O. Fès, O. Sebou }\end{array}$ & 1 & 1 & 16 & 10 & 5 & 33 \\
\hline O. Oum-er-Rbia & 4 & 1 & 10 & 1 & 2 & 18 \\
\hline $\begin{array}{l}\text { Lacs Agelman + } \\
\text { El Makhazine }\end{array}$ & & & 1 & 2 & & 3 \\
\hline $\begin{array}{l}\text { Total } \\
\%\end{array}$ & $\begin{array}{c}16 \\
11,9\end{array}$ & $\begin{array}{l}8 \\
6\end{array}$ & $\begin{array}{c}42 \\
31,3\end{array}$ & $\begin{array}{c}45 \\
33,6\end{array}$ & $\begin{array}{c}23 \\
17,2\end{array}$ & $\begin{array}{l}134 \\
100\end{array}$ \\
\hline
\end{tabular}

Notre étude, essentiellement dans les milieux courants arrosant les villes de Fès, Meknès, Marrakech, Khenifra, nous a permis de porter à 134 le nombre des espèces recensées sur le territoire marocain (Tableau III). Elles se répartissent ainsi dans les principales sous-familles ou tribus : 16 Tanypodinae, 8 Diamesinae, 42 Orthocladinae, 45 Chironomini, 23 Tanytarsini.

Avec 33 espèces nouvellement citées, l'étude des Oueds autour de Fès s'est avérée la plus fructueuse.

Ces résultats constituent une appréciable contribution à la connaissance des Chironomidés d'Afrique du Nord et font de la faune chironomidienne du Maroc la mieux connue actuellement : en effet, 14 espèces sont seulement signalées d'Algérie (Reiss 1977) et quelque 75 espèces ont été récemment identifiées de Tunisie (Coll. Boumaïza, Laville dét., données non publiées).

A partir de ce premier bilan, un premier état biogéographique peut être envisagé par comparaison avec lès faunes méditerranéenne et éthiopienne. Le tableau IV récapitule les espèces marocaines communes avec ces deux régions et dont les listes ont été dénombrées :

- pour la région méditerranéenne, dans les travaux de Reiss (1977, 1985, 1986), Moubayed \& Laville (1983) :

- pour la région éthiopienne, dans les travaux de Freeman (1955-1958) et Dejoux (1973).

Ainsi, 58 à $65 \%$ d'espèces sont communes avec la faune de méditerranée orientale ou occidentale et $8,2 \%$ seulement sont des espèces d'origine éthiopienne.

\begin{tabular}{lrrrr}
\hline & $\begin{array}{c}\text { Maroc Région mediterranéenne } \\
\text { orientale }\end{array}$ & $\begin{array}{c}\text { Région } \\
\text { occidentale }\end{array}$ \\
& & & ethiopienne \\
\hline Tanypodinae & 16 & 10 & 8 & 3 \\
Diamesinae & 8 & 2 & 5 & 0 \\
Orthocladiinae & 42 & 33 & 24 & 0 \\
Chironomini & 45 & 29 & 26 & 5 \\
Tanytarsini & 23 & 11 & 15 & 3 \\
Total & 134 & 85 & 78 & 11 \\
\hline
\end{tabular}

Les 11 espèces de la région éthiopienne sont les suivantes :

Ablabemyia ebbae

Paramerina mauritanica

Tanypus brevipalpis

Chironomus calipterus

Dicrotendipes pilosimanus

Leptochironomus deribae

Polypedilum aegyptium

Polypedilum (Pentapedilum) n. sp. (Marokko) (Reiss

1977)

Tanytarsus brevipalpis

Tanytarsus horni

Tanytarsus n. sp. (Marokko) (Reiss 1977)

Signalons par ailleurs que 6 espèces ne sont jusqu'ici connues que d'Afrique du Nord:
Rheopelopia n. sp.
: Maroc (Reiss 1977)
Telopelopia maroccana: Maroc
Rheomus alatus : Maroc
Rheomus yahiae : Maroc + Tunisie.
Stempellina almi : Maroc + Algérie.
Virgatanytarsus ansatus: Maroc + Algérie. 


\section{Travaux cltés}

Albu (P.). 1980. - Indexul sistematic al speculor din Romania. Ordinul Diptera familia Chironomidae Meigen sub familia Chironominae. In Fauna Republici socialiste Romania., vol. XI., fasc. 13:82-307.

Dejoux (C.). 1973, - Données faunistiques nouvelles concernant les Chironomides (Diptères, Nématocères) de la région éthiopienne. Cah. O.R.S.T.O.M., série Hydrobiol. VII (2) : 77-93.

1. - Fittkau (E.J.). 1962. - Die Tanypodinae (Diptera, Chironomidae). Abh. Larvalsyst. Insekter, 6: 1453.

Fittkau (E.J.) \& Reiss (F.). 1978. - Chironomidae (Diptera) In Illies J. (Ed.) Limnofauna Euporaea 2. Aufl. 404-440, G. Fischer, Stuttgart.

Freeman (P.). 1955. - A study of the Chironomidae (Dip tera) of Africa south of the Sahara. I. Bull. Br. Mus. nat. Hist. Ent., $4: 1-67$.

Freeman (P.). 1956. - A study of the Chironomidae (Dip tera) of Africa south of the Sahara II. Bull. Br. Mus. nat. Hist., Ent., 4 : 287-368.

Freeman (P.). 1958. - A study of the Chironomidae (Diptera) of Africa south of the Sahara IV. Bull. Br. Mus. nat. Hist. Ent., $6: 263-363$.

2. - Kügler (J.) \& Reiss (F.). 1973. - Die triangularis-Gruppe der Gattung Tanytarsus v.d.w. (Chironomidae, Diptera). Ent. Tidskr., $94:$ 59-82.

Laville (H.) Reiss (F.), sous presse. - Rheomus, un nouveau genre du complexe Hamischia avec deux nouvelles especes d'Afrique du Nord (Diptera, Chironomidae). Spixiano.

3. - Kügler (J.) \& Wool (D.). 1968 . - Chironomidae (Diptera) from the Hula Nature Preserve Israel. Ann. zood. fenn. 5 : 76-83.

4. - Lehmann (J.). 1970. - Revision der europäischen Arten (Imagines $\sigma \circ$ und Puppen $\sigma \sigma$ der Gattung Rheotonytarsus Bause (Dipt, Chironomidae). Zool. Anz., $185: 345.378$.

5. - Lehmann (J+). 1981. - Chironomidae (Diptera) aus Fliessgewässern Zentralafrikas. Il Teil : Die Region um Kisangani, Zentralzaire. Spixiana Suppl., 5 ; 3-85.

Moubayed (Z.) \& Laville (H.). 1983. - Les Chironomidés (Diptera) du Liban. I. Premier inventaire faunistique. Annis Limnol., 19 (3) : 219-228.

6. - Murray (D.A.). 1980. - Telopelopia maroccana sp. n. a second paleartic species of Telopelopia Roback (Diptera, Chironomidae). Acto Universitatis Cakolinae. Ser. Biologica, (1978), 12: 151.156.
7. - Ramdani (M.) \& Tourenq (J.N.). 1982. - Contribution a l'étude faunistique de la Merja de Sidi Bou Ghaba, Chironomides (Diptères). Bull. Inst. Scient. Rabat. $n^{\circ} 6: 179-185$.

8. - Reiss (F.). 1977. - Verbreitungsmuster bei paläarktischen Chironomidenarten (Diptera, Chironomidae). Spixiana, 1 : 85-97.

9. - Reiss (F.). 1985. - Contribution to the zoogeography of the turkisch Chironomidae (Diptera), Isr. J. Entomol., 19: 161-170.

to. - Reiss (F.). 1986. - Ein Beitrag zur Chironomidenfauna Syriens (Diptera, Chironomidae). Entomotauna Zeitschrift für Entomologie, 7 (11): $153-168$.

11. - Reiss (F.) \& Fittkau (E.J.). 1971. - Taxonomie und Okologie europaisch verbreiteter Tanytarsus-Arten (Chironomidae. Diptera). Arch. Hydrobiol. Suppl., $40: 75-200$.

12. - Reiss (F.) \& Sawedal (L). 1981. - Keys to males and pupae of the Paleartic (excl. Japan) Poratanytersus Thienemann \& Bause. 1913, n. comb., with descriptions of three new species (Diptera: Chironomidae). Ent. scand. Suppl., 15: 73-104.

13. - Reiss (F.) \& Schurch (M.). 1984. - Virgatanytarsus ansa ius n. sp. aus Mitteleuropa und Nordafrika. Spixiana, 7 (3): 319-322.

14. - Saether (O.A.). 1968. - Chironomids of the Finse Area Norway. with special reference to their distribution in a glacier brook. Arch. Hydrobiol., 64: 426-483.

15. - Serra-Tosio (B.). 1967. - Taxonomie et écologie des Diamesa du groupe latitarsis (Diptera, Chironomidae). Trav. Lab. Hydrobiol. Piscic. Univ. Gremoble, 57.58: 65-91.

16. - Serra-Tosio (B.), 1973. - Ecologie el biogéographie des Diamesinae d'Europe (Diptera, Chironomidae). Trav. Lab. Hydrobiot. Grenoble, $63: 5-175$.

17. - Serra-Tosio (B.). 1983. - Données biogéographiques nouvelles sur les Diamesinae des montagnes d'Asie et d'Afrique. $108^{\circ}$ Congrès national des Sociétés savantes, Grenoble, Sciences, fasc. II : 257-268.

18. - Vaillant (F.7. 1955. - Recherches sur la faune madicole de France, de Corse et d'Afrique du Nord. Thèse Fac. Scien. Univ. Paris. A-2744, $n^{\circ} 3617: 258 \mathrm{p}$.

Vilchez-Quero (A.) \& Laville (H.). 1987. - Eukiefferiella bedmari $n$. sp. nouvelle espèce à répartition méditerranéenne (Diptera, Chironomidae). Annls Limnol., 23 (3): 209-216.

19. - Wülker (W.). 1959. - Zur Kenntnis der Gattung Psectrocladius Kieff. (Dipt. Chironom.), Individuelle Variabilität, Grenzen und Möglichkeiten der Artentrennung, Okologie und Verbreitung. Arch. Hydrobiol. Suppl. $24: 1-66$. 